\title{
Heart transplantation from a dermatomyositis donor: Case report and review
}

\author{
Vincent I Lau ${ }^{1}$, Nakul Sharma ${ }^{2}$, Michael CY Chan ${ }^{3}$, Wayne Tymchak ${ }^{3}$ \\ 1. University of Alberta, Faculty of Internal Medicine, Edmonton, AB, Canada. 2. University of Calgary Libin, Cardiovascular \\ Institute of Alberta, Faculty of Medicine, Division of Cardiology, Calgary, AB, Canada. 3. University of Alberta Mazankowski, \\ Heart Institute, Division of Cardiology, Edmonton, AB, Canada
}

Correspondence: Vincent Lau. Address: University of Alberta, Faculty of Internal Medicine, 8410-112 Street, Edmonton, Alberta, T6G 2B7, Canada. Email: vinceissaclau@gmail.com

Received: September 1, 2015

Accepted: October 18, 2015

Online Published: October 20, 2015

DOI : $10.5430 /$ crim.v2n4p59

URL: http://dx.doi.org/10.5430/crim.v2n4p59

\begin{abstract}
Dermatomyositis is known to have sequelae outside of dermatological and musculoskeletal findings. These can also include pulmonary manifestations in the form of interstitial lung disease (ILD). Cardiac manifestations can include: myopericarditis, dysrhythmias and congestive heart failure. There are even patients with dermatomyositis who have required ventricular assist devices, pacemakers, implantable cardiac devices and even cardiac transplantation. However, we report the case of a patient with dermatomyositis with associated ILD who required lung transplantation who subsequently became a cardiac donor himself after a catastrophic neurological event during his pulmonary deterioration and cardiac work-up. With no prior literature, to our knowledge, this is the first published description of usage of a dermatomyositis donor in with successful cardiac transplantation without post-operative sequelae of dermatomyosits cardiac manifestations.
\end{abstract}

\section{Keywords}

Dermatomyositis, Cardiac transplantation, Interstitial lung Disease

\section{I ntroduction}

Dermatomyositis is characterized by immune-complex disposition into blood vessels with complement activation which is primarily diagnosed through the presence of dermatological and musculoskeletal findings. Such dermatological findings include: Gottron's papules (pathognomonic), helitrope rash, shawl sign and flagellated erythema. Musculoskeletal findings include: progressive proximal muscle weakness which have associated creatinine kinase elevation. Further investigations include anti-Jo1, anti-Mi-2 antibodies, electromyography (EMG) studies showing spontaneous activity, decreased amplitude and polyphasic potentials with contraction, as well as muscle biopsy which shows muscle fiber necrosis with perimysial and perivascular inflammation (B \& CD4 T-Cells), with complement in intramuscular blood vessels ${ }^{[1,2]}$.

There are also possible pulmonary manifestations of dermatomyositis in the form of primarily interstitial lung disease (pulmonary fibrosis) which may require lung transplantation. Cardiac manifestations can include: myopericarditis, conduction defects, dysrhythmias and congestive heart failure. There are even patients with dermatomyositis who have 
required pacemakers, implantable cardiac devices, ventricular assist devices and also eventual cardiac transplantation themselves ${ }^{[1,3]}$.

We report the case of a patient with interstitial lung disease (ILD) secondary to underlying dermatomyositis who was listed initially for lung transplantation. Through eventual deterioration with hypoxemic respiratory failure requiring venousvenous extracorporeal membrane oxygenation (ECMO) and part of his transplant work-up necessitating a coronary angiogram, the dermatomyositis patient eventually had a catastrophic neurological event with cerebral edema and tonsillar herniation causing brain death. This potential lung transplantation recipient then became a donor for heart transplantation procurement and the subsequent recipient currently has no post-transplantation dermatomyosits cardiac manifestations.

\section{Case report}

A 45-year-old male, who was previously healthy (except for a remote 15 pack year smoking history, quitting 15 years ago), presented with progressive dyspnea and associated arthralgias, myalagis and skin rashes over a one-month period. He had no cough, sputum production, hemoptysis with no associated fevers, chills, night sweats or weight loss. He eventually presented to hospital with worsening hypoxia requiring 4 litres of oxygen to maintain oxygen saturation above $90 \%$. The patient had no prior cardiac disease (specifically no coronary artery disease, dysrhythmias, heart failure symptoms, valvular disease) and no other cardiac risk factors (no diabetes, hypertension, dyslipidemia, cardiac family history).

Initial work-up for this patient which included blood cultures, sputum culture and gram stain with were all negative. His chest X-ray showed peri-hilar reticular-nodular interstitial disease and a follow-up computerized tomography (CT) chest showed no pulmonary embolism but had bilateral multi-focal pulmonary nodular parenchymal changes (with early cavitation) with associated bilateral lymphadenopathy (see Figure 1). A bronchoscopy was sought for bronchoalveolar lavage which revealed negative cultures for bacteria, fungus, acid-fast bacilli (AFB), respiratory viruses including influenza, and pneumocystis species. A thoracics consultation yielded a video-assisted thoracoscopy surgery in which a lung biopsy was done showing interstitial lung disease of bronchiolitis obliterans with organizing pneumonia (BOOP) with lymphoid nodules in the setting of probably collagen vascular disease. A rheumatology consult was obtained while the patient was in the intensive care unit (ICU). The patient had a rising creatinine kinase level to approximately $400 \mathrm{IU} / \mathrm{L}$. Initial serological work-up performed by ICU was negative for ANA, ANCA, RF, anti-CCP, anti-Jo1, anti-Scl-70 and ENA (which includes an anti-SS-A, anti-SS-B, RNP, anti-Sm). Quantitative immunoglobulins were also normal. However, on the basis of physical examination, the patient had pathognomonic Gottron's papules on both hands, and a diagnosis of dermatomyositis with associated interstitial lung disease was made (BOOP - dermatomyositis ossificans). Despite initial doses of intravenous immunoglobulin (IVIg), the patient's hypoxia continued to deteriorate, and Optiflow was required (50 litres, $\mathrm{FiO}_{2}$ 100\%). His final arterial blood gas prior to intubation on 100\% oxygen was as follows: $\mathrm{pH}$ 7.44, $\mathrm{pCO}_{2} 34 \mathrm{mmHg}, \mathrm{pO}_{2} 64 \mathrm{mmHg}, \mathrm{HCO}_{3} 20 \mathrm{mmol} / \mathrm{L}$, for an oxygen saturation of 93\%. Further respiratory decompensation necessitated intubation and mechanical ventilation while in ICU and consultations to the pulmonary transplant team (for lung transplant) as well as cardio-thoracic surgery (for ECMO) were sought after. Meanwhile, rheumatology started the patient on intravenous (IV) cyclophosphamide $1 \mathrm{~g}$ and pulsed IV methylprednisone $125 \mathrm{mg}$ daily to try to slow the progression of ILD.

Eventually, the cardio-thoracic surgeon initiated venous-venous (V-V) ECMO through an Avalon Elite bicaval dual lumen cannulae (Avalon Laboratories, Rancho Dominguez, CA) into the right internal jugular vein in order to oxygenate this patient in hypoxic respiratory failure. The V-V ECMO catheter was in good position with its output cannulae jet directed towards the tricuspid valve. After initiation of V-V ECMO, telemetry started noting ST-elevation which was confirmed on ECG. The patient was rushed urgently to the coronary catheterization laboratory, where an urgent coronary angiogram was performed. This revealed no concomitant coronary artery disease to explain the ST-elevation. Following this, the patient was transported to the cardiovascular ICU (CVICU). 
Upon arrival, it was noted that the patient had bilateral non-reactive dilated pupils and was sent urgently down for a CT scan of his head, which showed gross cerebral and cerebellar edema with loss of grey-white differentiation and effacement of basal cisterns and ventricular systems. But most important, it showed tonsillar herniation with brainstem compression, likely secondary to either hypoxic ischemic encephalopathy or venous congestion secondary to institution of V-V ECMO.

Figure 1. Initial CT chest at presentation: ground-glass interstitial lung disease (bilateral multi-focal pulmonary nodular parenchymal changes)

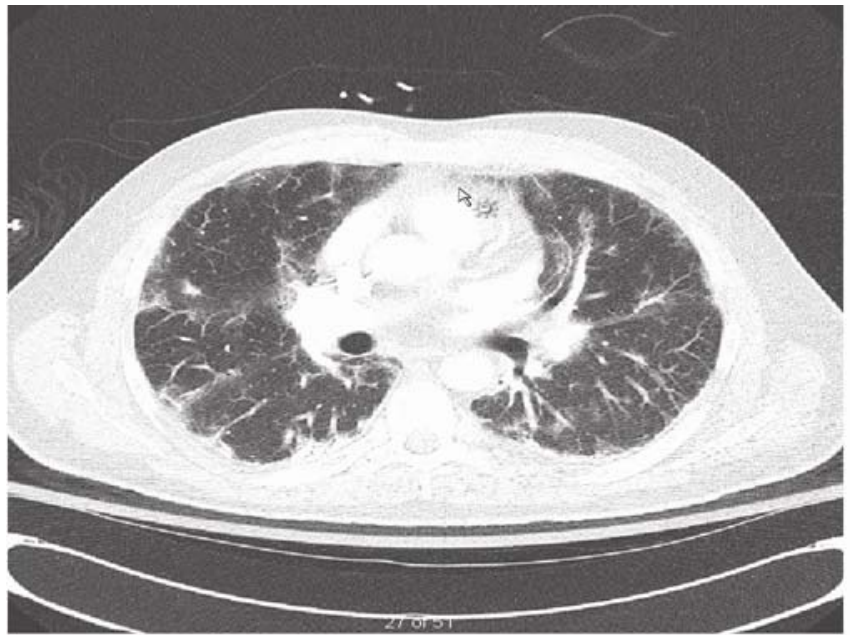

After letting know the family of this catastrophic neurological event in the patient, the cardiac transplant team was notified that this patient with underlying dermatomyositis could potentially act as a transplant donor himself. Given the lack of time for organ procurement, a cardiac biopsy was unfortunately not performed to see if there was any cardiac dematomyositis involvement. However, the patient still had a functionally normal heart based on prior transplant workup (see Figure 2): normal electrocardiogram (ECG) and 24 hour telemetry, normal echocardiogram with normal left ventricular size ejection fraction $(\mathrm{LVEF})>60 \%$ and size without diastolic dysfunction or hypertrophy (wall thickness $0.9 \mathrm{~cm}$ ), with normal right ventricular size and function, normal atria and normal functioning valves without significant regurgitation - mild tricuspid regurgitation - and no pericardial disease and normal pulmonary pressures) and pre-operative coronary angiogram. It was decided among the cardiac transplant team that the patient's heart would still be suitable for transplantation given that immunosuppression would ultimately be the overall treatment for cardiac dermatomyositis anyways, which the recipient would receive anyways for the transplantation Furthermore, a cardiac MRI showed no fibrosis burden related to dematomyositis.

A status 4 cardiac recipient (highest priority on the transplant list) was chosen. This patient was status 4 secondary to high allosensitization with high panel reactive antibodies from prior transfusions and had dilated cardiomyopathy with a prior LVEF of $10 \%$ on a left ventricular assist device (LVAD) with recurrent pump site pocket infections which required urgent explantation given active sepsis ongoing in this patient. Given that the donor's heart would likely not go to other recipient and that this current recipient was in dire need of a heart transplant, the cardiac team approached the family, who agreed for their family member to become a donor.

Organ procurement of the donor patient's heart was done and an explantation of the recipient LVAD with successful orthotropic heart transplantation was completed. As of this publication, the recipient is doing well post-transplantation with no evidence of dermatomyositis cardiac complications. His current anti-rejection cardiac transplant regime includes mycophenalate mofetil 1g PO BID and Tacrolimus $1 \mathrm{mg}$ PO BID. He is currently off Prednisone. His follow-up endomyocardial biopsies show no evidence of rejection (ISHLT ACR0R/AMR 0). His transthoracic echocardiogram (TTE) shows normal valves and normal LVEF. His ECG also shows no signs of arrhythmias or heart block. 


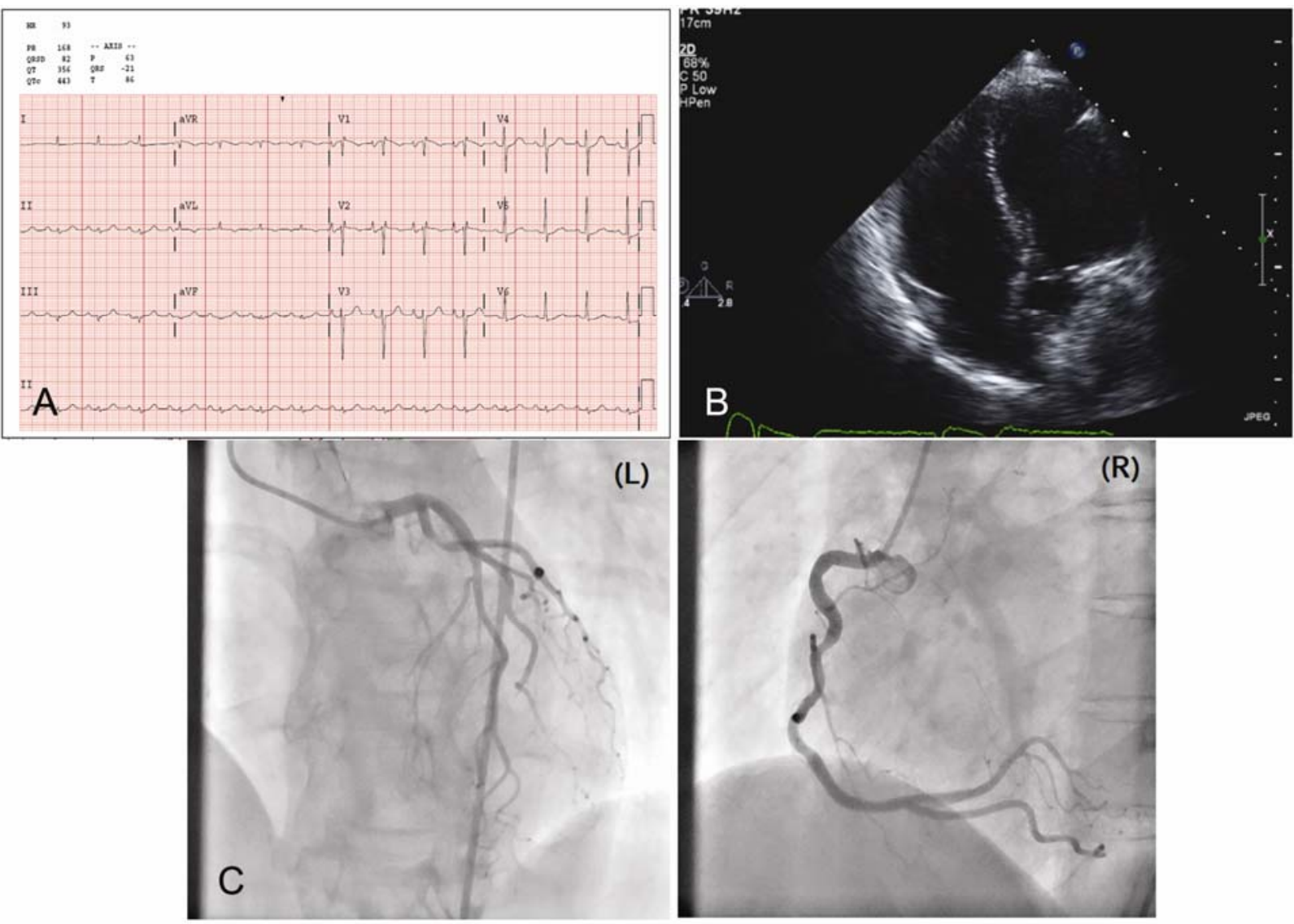

Figure 2. Pre-operative donor cardiac transplant work-up. Panel A: pre-transplant electrocardiogram; Panel B: pre-transplant echocardiogram; Panel C: pre-transplant

Note. Panel A: Pre-transplant electrocardiogram (ECG); Panel B: Pre-transplant echocardiogram with apical 4 chamber view seen below (normal left ventricular function, normal left ventricular size without diastolic dysfunction or hypertrophy (wall thickness $0.9 \mathrm{~cm}$ ), with normal right ventricular size and function, normal atria and normal functioning valves without significant regurgitation - only mild tricuspid regurgitation - and no pericardial disease and normal pulmonary pressures); Panel C: Pre-transplant coronary angiogram. Image on the left $(\mathrm{L})$ is the left coronary system, while the image on the right (R) is of the right coronary artery, neither of which have any significant coronary artery stenoses.

\section{Discussion}

It is well known that there can be cardiac manifestations of inflammatory myositis (i.e. dermatomyositis). This was an unfortunate case of a patient with underlying dermatomyositis (DM) patient with sequelae of ILD, with hypoxemic respiratory failure requiring a lung transplant. However, because of his rapid decline, he required V-V ECMO and had diffuse cerebral edema, tonsillar herniation causing brain stem compression and neurological brain death on nuclear medicine imaging. Despite this catastrophic neurological outcome while on ECMO of this dematomyositis patient, he did become a donor for orthotopic cardiac transplantation for another patient with dilated cardiomyopathy on an LVAD.

The question during this entire work-up and discussion was would a patient with underlying dermatomyositis with no current cardiac manifestations (based on normal ECG, telemetry, coronary angiogram, echocardiogram and MRI heart without fibrosis) be a good candidate for orthotopic cardiac transplant donation? Have there been any case reports of patients with underlying dermatomyositis or other inflammatory myosites who have been cardiac organ donors? If so, what have been the overall outcomes? Was there any difference in their management in terms of immunosuppression or treatment of underlying dermatomyositis cardiac manifestations?

A review of the literature was performed prior to orthotopic cardiac transplantation of this dermatomyositis (DM) patient in 2012, and repeat review (MEDLINE, key word: myositis and cardiac transplantation) was done at the time of this 
publication. As of May 2014, there have been only 4 case reports of inflammatory myositis and cardiac transplantation. Of these case reports, all of them describe the patient with inflammatory myositis as the recipient of cardiac transplantation: one case of toxoplasmosis-induced cardiac polymyositis post-transplant and one case of Cryptococcal cardiac myositis in an HIV+ patient post-cardiac transplant. The other two cases were of patients who had underlying polymyositis and cardiac manifestations of congestive heart failure requiring eventual heart transplantation recipients. There have been no reported cases of a myositis patient as a donor.

Therefore, our case is unique in that there is no prior literature or case reports of a donor patient with dermatomyositis (with no cardiac manifestations of DM) at the time of heart procurement and donation that has had as successful heart transplant into a recipient. As mentioned previously, the cardiac work-up was quite extensive in addition to the standard workup (ECG, echocardiogram and coronary angiogram) pre-transplant for donors - which were all normal and negative for cardiac dermatomyositis involvement. Since cardiac transplantation requires immunosuppression to prevent organ rejection, and that the overall treatment for possible cardiac dematomyositis was immunosuppression, we felt we would ultimately suppress cardiac dematomyositis anyways. Furthermore, because of the controversy of this particular organ as suitable organ, plus the urgency of our dire status 4 cardiac recipient (high allosensitization and active sepsis from LVAD pocket infections). This all led to the expansion of the donor criteria, making our patient eligible as a donor. Since there have been no case reports to suggest that a DM cardiac donor transplant was contraindicated, therefore we went ahead with donation. At 2 year follow-up, the recipient has no signs of rejection or cardiac manifestations of DM based on ECG, TTE and cardiac biopsy.

During transplantation discussion for this patient and his potential recipient, Rheumatology noted that given the treatment for underlying DM is immunosuppression (corticosteroids and azathioprine). Therefore, since the patient was already going to be on immunosuppression for anti-rejection of the orthotopic heart transplant, it was thought that DM manifestations would be less likely in the transplanted heart. The recipient's current regime of tacrolimus (calcineurin inhibitor) and mycophenalate mofetil (Cellcept) are even more potent immunosuppressants compared to corticosteroids and azathioprine normally used to control DM.

In conclusion, we believe that patients with underlying inflammatory myositis but no active cardiac manifestations can be suitable candidates for cardiac transplant donation. Immunosuppressant regimes for the recipient cardiac transplant would not have to differ as such given that treatment of underlying dermatomyositis also includes immunosuppression.

\section{References}

[1] Dalakas MC, Hohlfed R. Polymyositis and dermatomyositis. Lancet. 2003; 362: 971-82. http://dx.doi.org/10.1016/S0140-6736(03)14368-1

[2] Bohan A, Peter JB. Polymyosiitis and dermatomyositis. NEJM. 1975; 292: 334-47. PMid:1090839 http://dx.doi.org/10.1056/NEJM197502132920706

[3] Lundberg IE. The heart in dermatomyositis and polymyositis. Rheumatology. 2006; 45: iv18-iv21. PMid:16980718 http://dx.doi.org/10.1093/rheumatology/kel311

[4] Afzal A, Higgins R, Philbin E. Heart transplant for dilated cardiomyopathy associated with polymyositis. Heart. $1999 ; 82:$ e4. PMid:10490578 http://dx.doi.org/10.1136/hrt.82.4.e4

[5] Morrissey RP, Rana JS, Luthringer DJ, et al. Case of fulminant giant-cell myocarditis associated with polymyositis, treated with a biventricular assist device and subsequent heart transplantation. Heart \& Lung. 2011; 40: 340-5. PMid:21419487 http://dx.doi.org/10.1016/j.hrtlng.2010.06.003

[6] O'Neill KM, Ormsby AH, Prayson RA. Cryptococcal myositis: a case report and review of the literature. Pathology. 1998; 30: 316-7. PMid:9770201 http://dx.doi.org/10.1080/00313029800169526

[7] Cuturic M, Hayat GR, Vogler CA, et al. Toxoplasmic polymyositis revisited: case report and review of literature. Neuromuscular Disorders. 1997; 7: 390-6. http://dx.doi.org/10.1016/S0960-8966(97)00098-9 